\title{
EXIT-Chart Aided Hybrid Multiuser Detector Design for Frequency-Domain-Spread Chip-Interleaved MC-CDMA
}

\author{
Lei Xu, Rong Zhang, Sheng Chen and ${ }^{1}$ Lajos Hanzo \\ School of ECS., Univ. of Southampton, SO17 1BJ, UK. \\ Tel: +44-23-80-593 125, Fax: +44-23-80-593 045 \\ Email: $\{1 \mathrm{~h}\} @$ ecs.soton.ac.uk, http://www-mobile.ecs.soton.ac.uk
}

\begin{abstract}
With the advent of EXtrinsic Information Transfer (EXIT) charts, we are capable of analyzing, predicting and visually comparing the convergence behaviours of different turbo Multi-User Detectector (MUD)s. The different MUDs have diverse EXIT characteristics and hence their superposition allows us to create a combined EXIT curve, which closely matches that of the channel decoder. Hence a near-capacity operation is facilitated by combining the benifits of different MUDs and therefore to create a superior MUD. Thus in this contribution, we propose a novel hybrid MUD combining scheme, which combines the advantages of a high performance and low complexity in form of an advanced hybrid MUD solution. The transmitted bits are unknown at the receiver, hence it is not feasible to directly evaluate the mutual information gain of the iterative MUD in consecutive iterations, hence we propose a realistic algorithm for estimating this mutual information gain, which is then used for activating the most appropriate constituent MUD as and when it is necessary. The constituent MUDs are the Matched Filter (MF) based Soft Interference Cancellation (SoIC) and the optimum Bayesian MUDs, which are invoked in the scenario of Frequency-Domain-Spread ChipInterleaved (FDSCI) Multiple Carrier Code Division Multiple Access (MC-CDMA). The resultant hybrid MUD is capable of outperforming both the MF-SoIC and Bayesian turbo MUDs in the terms of the attainable complexity and Bit-Error-Rate (BER) performance.
\end{abstract}

\section{INTRODUCTION}

The Frequency-Domain-Spread Chip-Interleaved Multiple Carrier Code Division Multiple Access (FDSCI MC-CDMA) scheme considered may be interpreted as the multi-carrier version of the Interleave Division Multiple Access (IDMA) philosophy developed by Ping et. al. [1]. The IDMA scheme exchanges the classic position of DSspreading and interleaving employed in traditional CDMA systems, leading to chip-interleaving instead of bit-interleaving. Hence the different users are distinguished by their unique user-specific interleavers $\pi_{k}$, as seen in Fig. 1.

The IDMA receiver of [1] employs turbo-style iterative joint Matched Filter based Soft Interference Cancellation (MF-SoIC) aided Multi-User Detectector (MUD) combined with channel decoding, where the MUD's front-end processing is carried out on a chipby-chip basis. However, MF based detection constitutes a singleuser approach, which has a limited ability to exploit any extrinsic information and hence its performance is interference-limited. In contrast to the low-complexity MF-SoIC MUD, the optimal Bayesian MUD imposes an exponentially increasing complexity as a function of the number of users.

EXtrinsic Information Transfer (EXIT) charts [2] are capable of analyzing the convergence characteristic of concatenated turbo components and constitute a convenient semi-analytical performance visualization technique. More explicitly, EXIT charts evaluate the mutual information of the extrinsic output and a priori input of the concatenated decoder components corresponding to the associated bits $s$. The resultant extrinsic and a priori information exchange between the iterative Soft-In Soft-Out (SISO) blocks is then portrayed, as detailed in [2]. Thus the different mutual information gain of the MUDs serially concatenated with a SISO channel decoder may

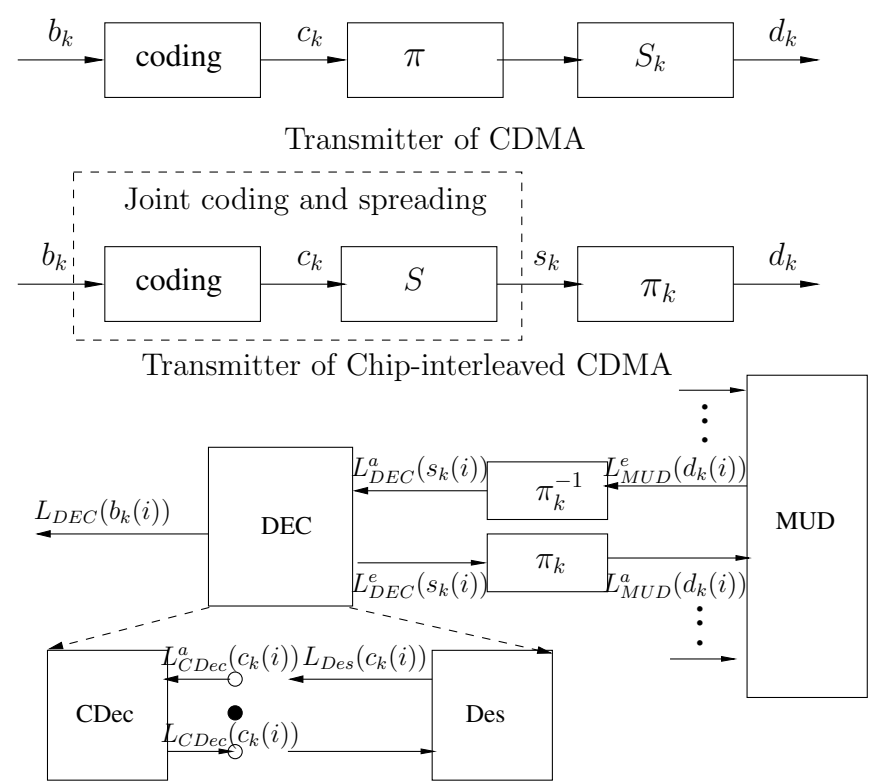

Iterative Receiver of chip-interleaved CDMA

Fig. 1. The transceiver of chip-interleaved CDMA

be visualized at each step of the iterative detection process in the EXIT chart. Therefore, an advanced scheme combining the benifits of different MUDs may be created.

Hence the novelty of this contribution is that we propose a hybrid MUD, which activates different MUDs in different iterations by exploiting a low-complexity mutual information evluation technique. Specifically, the proposed hybrid scheme enables us to circumvent the performance limitations of the MF-SoIC MUD by exploiting the superior performance of the Bayesian MUD during a few MUD iterations only. This allows us to approach the optimum Bayesian MUD's performance at a modestly increased complexity in comparison to that of the MF-SoIC MUD. As a further novel feature, we propose an algorithm for the real-time monitoring of the actual mutual information exchange during the MUD process by evaluating the average of the LLR magnitudes at the output of the MUD.

The remainder of this contribution is organized as follows. In Section II, our system model is introduced, while the EXIT chart analysis is presented in Section III, which will be used in Section IV for studying a range of different turbo MUD strategies. Our system performance results and discussions will be presented in Section V, followed by our conclusions in Section VI.

\section{Transceiver Structure}

The upper part of Fig. 1 portrays the transmitter of the system. Assuming that BPSK modulation is adopted here, the $k$ th user's 
transmitted bit stream $\left\{b_{k}(i) ; i=1,2, \cdots, M\right\}$ is firstly channel encoded at a rate of $R=M / N_{c}$, yielding the encoded stream $\left\{c_{k}(i) ; i=1,2, \cdots, N_{c}\right\}$. Then the resultant channel encoded stream is Direct Sequence (DS) spread by a spreading code having a spreading gain $G$, which is common for all users, yielding the sequence $\left\{s_{k}(i) ; i=1,2, \cdots, N\right\}$. This sequence is then chip-interleaved employing a user-specific random chip-interleaver $\pi_{k}$, in order to form the chip stream $\left\{d_{k}(i) ; i=1,2, \cdots, N\right\}$. The resultant chip stream is then transmitted through the multiple access channel supporting $K$ users. This is in contrast to the concept of a classic CDMA system, where the interleaver is between the FEC coding and DS-spreading stages. The latter classic philosophy corresponds to bit-interleaving, which is also seen in the upper part of Fig. 1. Then each user's signal is OFDM modulated [3]. At the receiver, after OFDM demodulation the $u$-th subcarrier's received signal in the $i$-th OFDM symbol is denoted by

$$
y=\mathbf{H d}+\nu,
$$

where $\mathbf{H}$ is the $K$ dimensional Frequency Domain Channel Transfer Function (FDCHTF) vector corresponding to the $K$ users, while the $K$ users' data vector is denoted by $\mathbf{d}$. Furthermore, $\nu$ is the Additive White Gaussian Noise (AWGN) having a zero mean and a variance of $2 \sigma_{n}^{2}$.

\section{EXIT CHART ANALYSIS}

EXIT charts were proposed by ten Brink [2] for analyzing the convergence characteristic of turbo codes as a convenient visualization technique. This technique computes the mutual information of the output extrinsic and input a-priori components corresponding to the associated bits $s$ for each of the iterative SISO blocks. More specifically, the mutual information of the LLRs and the legitimate bipolar BPSK bits is given by [2]:

$$
\begin{aligned}
\mathbf{I}= & \frac{1}{2} \sum_{s \in\{+1,-1\}} \int_{-\infty}^{\infty} p_{L}(x \mid s) \\
& \cdot \log _{2}\left[\frac{2 p_{L}(x \mid s)}{p_{L}(x \mid+1)+p_{L}(x \mid-1)}\right] d x,
\end{aligned}
$$

where $p_{L}(x \mid s)$ is the conditional probability distribution of the LLRs. We can now substitute the conditional probability distribution of the extrinsic information $L^{e}$ and that of the a-priori information $L^{a}$ into Eq. 2 in order to derive the mutual information between either $\mathbf{I}_{E}$ or $\mathbf{I}_{A}$ and the legitimate bipolar bits, respectively. The required distribution of the LLRs in Eq.2 can be approximated by the experimentally generated LLR histogram or using the ubiquitous Gaussian distribution for modeling the LLR distribution.

\section{TURBo MUDs}

\section{A. MF-SoIC turbo MUD}

The receiver of Fig. 1 consists of a Soft-In-Soft-Out (SISO) MUD and a bank of $K$ individual SISO channel DECoders (DEC), where the soft information exchanged between the receiver components is constituted by the extrinsic Log-Likelihood Ratios (LLRs). At the $l$-th iteration, the MF outputs the extrinsic information $L_{M U D}^{e}\left(d_{k}\right)$ based on the channel's output, combined with the a priori informantion $L_{D E C}^{e}\left(d_{k}\right)$ provided by the SISO DEC. The soft estimates $\hat{d}_{k}$ of the $k$ th user's signal are represented as:

$$
\mathbf{E}\left(d_{k}\right)=\tanh \left(L_{D E C}^{e}\left(d_{k}\right) / 2\right),
$$

while their variance is given by:

$$
\mathbf{V}\left(d_{k}\right)=1-\mathbf{E}\left(d_{k}\right)^{2} .
$$

When considering user $k$ as the user of interest, we have:

$$
y=H_{k} d_{k}+\xi
$$

where $\xi=\sum_{j \neq k} H_{k} d_{k}+\nu$ denotes the interference plus noise. The soft estimates of $\xi$ can be represented as

$$
\mathbf{E}(\xi)=\sum_{j \neq k} H_{k} \mathbf{E}\left(d_{k}\right),
$$

while the corresponding variance is expressed as :

$$
V(\xi)=\sum_{j \neq k}\left|H_{k}\right|^{2} \mathbf{V}\left(d_{k}\right)+2 \sigma_{n}^{2}
$$

Assuming that the received signal is Gaussian distributed around the legitimate transmitted symbol values, the resultant extrinsic information $L_{M U D}^{e}\left(d_{k}\right)$ can be expressed as:

$$
L_{M U D}^{e}\left(d_{k}\right)=\frac{4 \mathcal{R}\left\{H_{k}^{*}(y-\mathbf{E}(\xi))\right\}}{V(\xi)},
$$

where $\mathcal{R}$ denotes the real part of a complex variable. The calculation of $\mathbf{E}(\xi)$ and $V(\xi)$ requires totally $4(K-3)$ additions and multiplications, when considering a single transmitted chip.

\section{B. Bayesian turbo MUD}

Following the first MUD iteration, the a priori information concerning the likelihood of all the legitimate $N_{b}=2^{K}$ number of BPSK modulated $K$-user bit sequences becomes available. The joint PDF of the antenna array's output $y$ and the transmitted chip stream $d_{k}^{(j)} \in\{ \pm 1\}, j \in 1, \ldots, N_{b}$ of user $k$ at the output of the channel encoder can be expressed as the superposition of all the conditional Gaussian PDFs positioned at the legitimate noiseless channel outputs corresponding to the transmitted symbols $d_{k}=+1$ and $d_{k}=-1$, multiplied by the $j$-th legitimate signal vector's probabilities, respectively, which can be expressed as [4]:

$$
P\left(y, d_{k}= \pm 1\right)=\sum_{\forall j: d_{k}^{(j)}= \pm 1} P\left(d^{(j)}\right) e^{\left(-\frac{\left(\left\|y-\bar{y}_{j}\right\|\right)^{2}}{2 \sigma_{n}^{2}}\right)} .
$$

The entire set of $N_{b}=2^{K}$ number of legitimate vectors of the $K$ users is partitioned into two subsets corresponding to $d_{k}=+1$ and $d_{k}=-1$. In Eq.(9), $\bar{y}_{j}, j \in 1, \ldots, N_{b}$ constitutes the noiseless received signal vectors, where the Gaussian PDFs seen in Eq.(9) are centered. The Euclidian distance measured from their noiseless center to the received signal vector is used as the metric of quantifying their corresponding probability. Furthermore, $P\left(d^{(j)}\right)$ is the a-priori information to be defined more explicitly below. All the other notations are defined as before.

Provided that the chips of all the $K$ users are independent, the probability $P\left(d^{(j)}\right)$ can be expressed as :

$$
P\left(d^{(j)}\right)=\prod_{k=1}^{K} P\left(d_{k}^{(j)}\right), j \in 1, \ldots, N_{b},
$$

where $P\left(d_{k}^{(j)}\right)$ represents the probabilities of either $P\left(d_{k}^{(j)}=1\right)$ or $P\left(d_{k}^{(j)}=0\right)$, depending on the $l$-th user's bit at the $j$-th bit position, $j \in 1, \ldots, N_{b}=2^{L}$ within the $K$-user transmitted chip vector, which is the a-priori information provided by the $k$-th user's SISO decoder.

Let us use the conditional likelihood of the received signal vector as that of the estimated bit decision concerning the $k$ th user's transmitted 


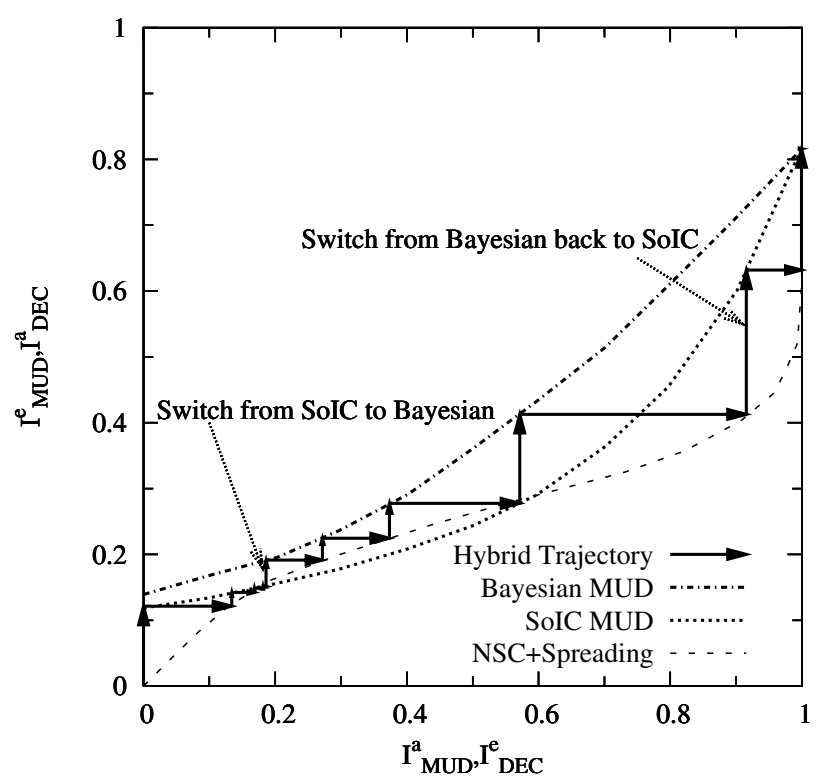

Fig. 2. Exit charts and simulated trajectories of the proposed hybrid MUD when supporting $K=12$ users at $E_{b} / N_{0}=10 d B$.

bit. Then we have:

$$
\begin{array}{r}
L_{M U D}^{e}\left(d_{k}\right)=\ln \frac{P\left(d_{k}=+1 \mid y\right)}{P\left(d_{k}=-1 \mid y\right)}-\ln \frac{P\left(d_{k}=+1\right)}{P\left(d_{k}=-1\right)} \\
=\ln \frac{\sum_{\forall j: d_{k}^{(j)}=+1}\left(\prod_{\forall l: l \neq k} P\left(d_{l}^{(j)}\right)\right) e^{\left(-\frac{\left(\left\|y-\bar{y}_{j}\right\|\right)^{2}}{2 \sigma_{n}^{2}}\right)}}{\sum_{\forall j: d_{k}^{(j)}=-1}\left(\prod_{\forall l: l \neq k} P\left(d_{l}^{(j)}\right)\right) e^{\left(-\frac{\left(\left\|y-\bar{y}_{j}\right\|\right)^{2}}{2 \sigma_{n}^{2}}\right)}} .
\end{array}
$$

This will lead to the maximum-likelihood solution. Furthermore, the complexity of the Bayesian turbo MUD can be reduced by employing the techniques proposed in [5]. The complexity of the above-mentioned Bayesian MUD is constituted by $2 * 2^{K}(2 K-1)$ additions and multiplications required for computing the legitimate channel output states $\bar{y}_{j}, j \in 1, \ldots, N_{b}$ and $7 * 2^{K}$ additions of the Gaussian PDFs, plus the $2^{K}$ exp function evaluations seen in Eq.(11), when considering a single transmitted chip. Hence, we can approximate the total complexity of the Bayesian MUD as $2^{K}(4 K+6)$ operations.

\section{Hybrid Turbo MUD Design}

Fig. 2 portrays the EXIT curves of both the SoIC aided MF and of the Bayesian MUDs, when supporting $K=12$ users with the aid of 4-chip Frequency-Domain (FD) spreading sequences at $E_{b} / N_{0}=10 \mathrm{~dB}$. At the abscissa value of unity, the EXIT curves of both constituent MUDs converge to the same point, coinciding with that recorded for a single user. This reveals that the multiple access interference (MAI) is completely eliminated by both the MUDs, provided that perfect a priori information is available. At the abscissa of zero, the Bayesian turbo MUD's EXIT curve exhibits a higher starting point than that of the MF-SoIC MUD and provides a significantly wider EXIT tunnel, albeit this is achieved at a cost of tremendously increased computational complexity. By contrast, the MF-SoIC MUD results in reduced $\mathbf{I}^{\mathbf{e}}$ MUD values in the EXIT chart at a significantly reduced complexity requirement. Generally, if there is an open tunnel between the EXIT curve of the MUD and that of the channel decoder, the MF-SoIC MUD would impose a lower complexity than the Bayesian turbo MUD, when aiming for

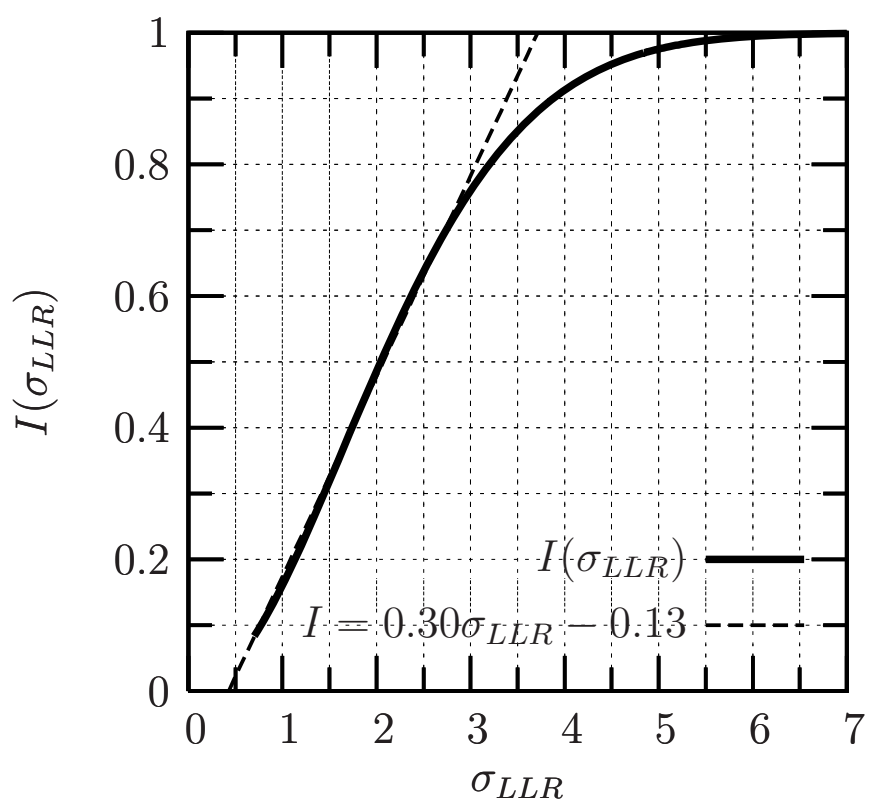

Fig. 3. The mutual information $I$ function of $\sigma_{L L R}$ and its linear approximation.

achieving a near-single-user performance. This implies that the MFSoIC MUD should be activated instead of the more complex Bayesian MUD, when the former is also capable of achieving a sufficiently high the iteration gain. However, at a low SNR value, there may not be a sufficiently widely open convergence tunnel when using the MFSoIC MUD, but it may still be open when using the Bayesian MUD, as seen in Fig.2.

Our novel proposition is to use the MF-SoIC MUD during the first a few iterations and subsequently to activate the Bayesian MUD for a few further iterations to avoid encountering the "bottleneck" region of the MF-SoIC MUD. Beyond the EXIT-chart's bottle-neck region we may then safely activate again the MF-SoIC MUD. As an explicit benefit, the proposed Hybrid MUD becomes capable of achieving a similar performance to that of the Bayesian MUD at a decreased complexity in comparison to the conventional method, since the Bayesian MUD is only activated during the critical MUD iterations. In order to determine the activation instant of the different MUDs, we have to monitor the mutual information gain between the MUDs and the channel decoder, as shown in Fig.2. If the mutual information improvements are monitored at the receiver, then it is intuitive that the MF-SoIC MUD should be actived when its EXIT curve is above that of the channel decoder, while the Bayesian MUD should be activated when the EXIT curve of the MF-SoIC MUD is below that of the channel decoder. However, in practice the knowledge of the mutual information is generally unknown at the receiver. Thus, we have to estimate of the extrinsic information's absolute value $E\left(\mid L_{M U D}^{e}\right) \mid$ generated by the MUD. Assuming that the LLRs obey a Gaussian distribution at the output of the MUD having a zero-mean and a variance of $\sigma_{L L R}$, the probability density function (PDF) of the extrinsic information's absolute value $\left|L_{M U D}^{e}\right|$ can be expresseed as:

$$
P\left(\left|L_{M U D}\right|\right)=\frac{2}{\sigma_{L L R} \sqrt{2 \pi}} \exp \left(-\frac{\left(L_{M U D}\right)^{2}}{2 \sigma_{L L R}^{2}}\right)
$$


Hence, we have:

$$
\begin{aligned}
E\left(\left|L_{M U D}^{e}\right|\right) & =\int_{0}^{+\infty} \frac{2 x}{\sigma_{L L R} \sqrt{2 \pi}} \exp \left(-\frac{x^{2}}{2 \sigma_{L L R}^{2}}\right) d x \\
& =\sqrt{\frac{2}{\pi}} \sigma_{L L R} .
\end{aligned}
$$

Similarly, Eq.2 can be rewritten as [2]:

$$
\mathbf{I}\left(\sigma_{\mathbf{L L R}}\right)=\mathbf{1}-\int_{-\infty}^{\infty} \frac{\mathrm{e}^{\left(\frac{-\left(\mathrm{x}-\sigma_{\mathrm{LLR}}^{2} / \mathbf{2}\right)^{2}}{2 \sigma_{\mathrm{LLR}}^{2}}\right)}}{\sqrt{\mathbf{2} \pi} \sigma_{\mathbf{L L R}}} \cdot \log _{2}\left(1+\mathbf{e}^{-\mathbf{x}}\right) \mathbf{d x}
$$

The function $\mathbf{I}\left(\sigma_{\mathbf{L L R}}\right)$ is monotonically increasing with the LLR variance $\sigma_{L L R}^{2}$, as shown in Fig.3. It is also seen in Fig.3 that the mutual information $\mathbf{I}\left(\sigma_{\mathbf{L L R}}\right)$ can be accurately approximated by a linear fuction in the range of $0.1<\mathbf{I}\left(\sigma_{\mathbf{L L R}}\right)<\mathbf{0 . 8}$ as:

$$
\mathrm{I}\left(\sigma_{\mathrm{LLR}}\right) \approx 0.3043 \cdot \sigma_{\mathrm{LLR}}-0.1303
$$

This range of $[0.1,0.8]$ typically includes the $\mathbf{I}_{\text {MUD }}^{\mathbf{e}}$ values experienced during the iterative process. Furthermore, using Eq.13, we arrive at:

$$
\mathbf{I}_{\text {MUD }}^{\mathrm{e}}\left(\mathrm{E}\left(\left|\mathrm{L}_{\mathrm{MUD}}^{\mathrm{e}}\right|\right)\right) \approx 0.3043 \sqrt{\frac{\pi}{2}} \mathbf{E}\left(\left|\mathbf{L}_{\mathrm{MUD}}^{\mathrm{e}}\right|\right)-0.1303,
$$

where $\mathbf{I}_{\text {MUD }}^{\mathrm{e}} \in[\mathbf{0 . 1}, \mathbf{0 . 8}]$. Hence, we can contrive a low-complexity scheme of estimating the extrinsic mutual information at the output of the MUD based on Eq.16. More explicitly, the average value of $\left|L_{M U D}^{e}\right|$ within the transmission block is computed after each MUD iteration, yielding $E\left(\left|L_{M U D}^{e}\right|\right)$. Then, the corresponding value of the mutual information $\mathbf{I}_{\text {MUD }}^{\mathrm{e}}$ can be dervied using Eq.16. In order to construct our hybrid MUD, we record the extrinsic mutual information gain $G_{I}(l)$, namely that of the incremental value of $I_{M U D}^{e, l}$ during $l$-th iteration compared to $I_{M U D}^{e, l-1}$ of the previous $(l-1)$ th iteration, which is expressed as:

$$
\begin{aligned}
G_{I}(l) & =\mathbf{I}_{\text {MUD }}^{\mathbf{e}, 1}-\mathbf{I}_{\text {MUD }}^{\mathbf{e}, 1-1} \\
& \approx 0.3043 \sqrt{\frac{\pi}{2}}\left[E\left(\left|L_{M U D}^{e, l}\right|\right)-E\left(\left|L_{M U D}^{e, l-1}\right|\right)\right] .
\end{aligned}
$$

Initially we activate the MF-SoIC MUD, but once the extrinsic mutual information gain $G_{I}(l)$ of the $l$-th iteration becomes lower than an experientially determinated threshold of $G_{I}(l)<\delta=0.05$, and simultaneously the current $\mathbf{I}_{\text {SoIC }}^{\mathbf{e}, 1}$ value is below the experimentally determined value of $\mathbf{I}_{\text {SoIC }}^{\mathbf{e}}=\mathbf{0 . 6}$, then the more complex but more powerful Bayesian MUD is activated. This allows the MUD to traverse through the "bottleneck" region of $\mathbf{I}_{\text {SoIC }}^{\mathbf{e}} \in[\mathbf{0 . 1}, \mathbf{0 . 3}]$ seen in Fig.2. Subsequently, when the extrinsic mutual information gain $G_{I}(l)$ of the $l$-th iteration becomes higher than another experientially determinated threshold of $G_{I}(l)>\Delta=0.12$, then the lowcomplexity MF-SoIC MUD is reactivated for the sake of reducing the overall complexity. In order to avoid any potential failure to converge, the requirement of maintaining a sufficiently high extrinsic mutual information gain of $G_{I}(l)$ after switching to the MF-SoIC MUD should remain always valid. As and when this condition is not satisfied, the Bayesian MUD should be reactivated. In other words, the proposed hybrid MUD will activate the MF-SoIC MUD during the entire iterative MUD process, when the SNR value is sufficiently high. Nonetheless, it achieves a similar performance to that of the Bayesian MUD at a lower complexity, since it only activates the Bayesian MUD, when the MF-SoIC MUD would result in a BER floor. The complexity of the hybrid MUD is determinated by the complexity of the MF-SoIC MUD and that of the Bayesian MUD weighted by the corresponding number of iterations.

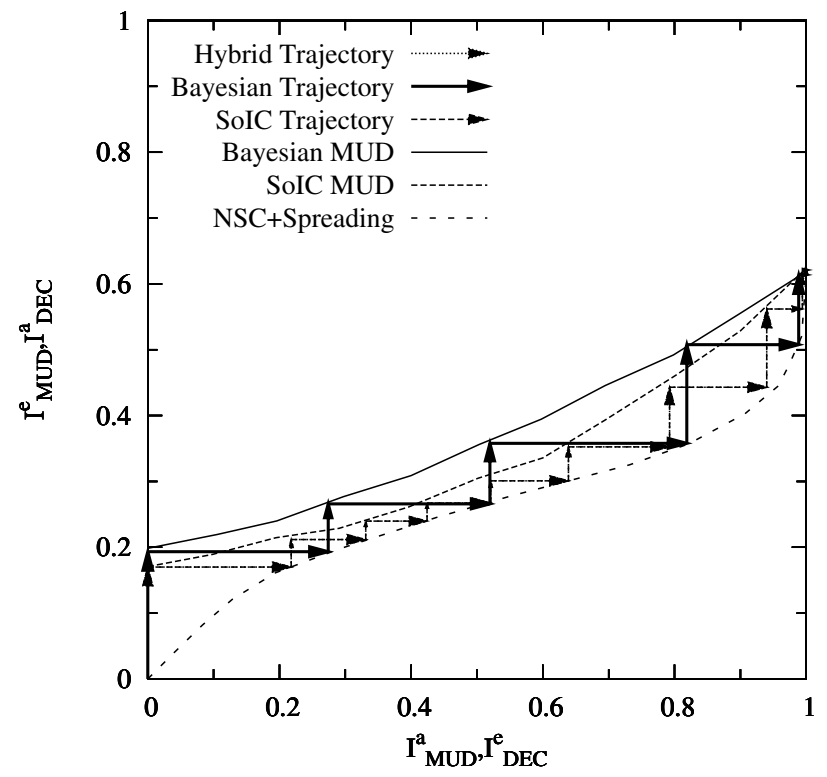

Fig. 4. Exit charts and simulated trajectories of the proposed hybrid MUD when supporting $K=8$ users at $E_{b} / N_{0}=6 d B$.

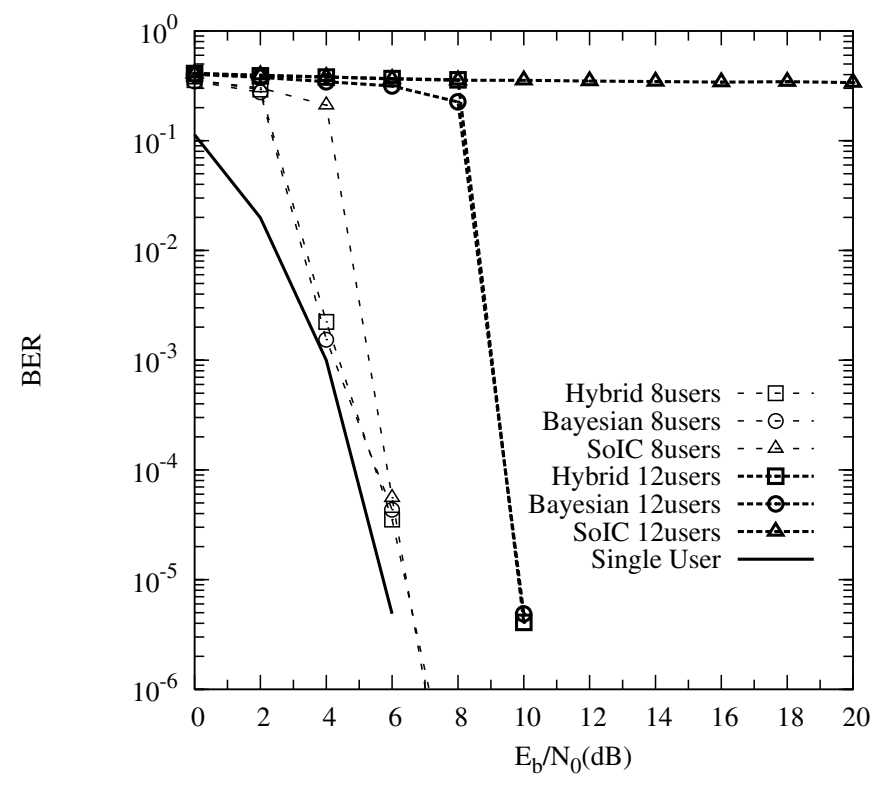

Fig. 5. BER performance of the proposed system

\begin{tabular}{|l|r|}
\hline MC-CDMA System Parameters & \\
\hline Number of users & 8,12 \\
\hline Number of receiver antennas & 1 \\
\hline Spreading factor & 2 \\
\hline $\begin{array}{l}\text { Channel impulse } \\
\text { response }\end{array}$ & 3-path SWATM \\
\hline Number of subcarriers & symbol-invariant [3] \\
\hline pength of cyclic prefix
\end{tabular}




\section{Simulation Results and Discussions}

In this section, the attainable performance of the proposed hybrid MUD is investigated in comparison to the two constituent turbo MUDs, namely that of the MF-SoIC MUD and the Bayesian MUD. Our system parameters are summarized in Table I. Each user has a different random interleaver. We will demonstrate that the proposed hybrid MUD strikes an attractive trade-off between the constituent MUDs employed as benchmarkers.

As seen in Fig.2, the simulation-based trajectory closely follows the EXIT curves of the receiver components. During the initial three iterations, the MF-SoIC MUD is activated. As the incremental extrinsic mutual information gain of each additional iteration is reduced, which is evaluated using the average absolute extrinsic LLR difference between the current and previous iterations with the aide of Eq.17, the Bayesian MUD is activated, in order to overcome the MF-SoIC MUD's EXIT tunnel constriction. When the consecutive iteration gains of the Bayesian MUD become sufficiently high again, the MF-SoIC MUD is reactivated in the interest of reducing the complexity imposed.

Fig. 4 shows both the EXIT curves and the associated simulationbased trajectories of all the MUDs concerned when supporing $K=8$ users at $E_{b} / N_{0}=6 d B$. At this particular SNR there is no need to activate the Bayesian MUD, since even the lower-complexity MFSoIC MUD is capable of maintaining an open EXIT tunnel, hence lending to an infinitesimally low BER.

Fig. 5 portrays the attainable BER performances of the MF-SoIC MUD, of the Bayesian MUD and of the proposed hybrid MUD as a function of the SNR as well as of the number of users supported. Observe in Fig.5 that at a high normalized user-load of $\gamma=K / S_{f}=$ $12 / 4$, the MF-SoIC MUD fails to converge, while the proposed hybrid MUD and the Bayesian MUD achieves an infinitesimally low BER for $E_{b} / N_{0}>10 d B$.

Explicitly, when supporting for example $K=12$ users with the aid of $S_{f}=4$-chip spreading sequences, the MF-SoIC benchmarker fails to provide an adequate performance, while the proposed hybrid MUD achieves an infinitesimally low Bit Error Rate (BER) for $E_{b} / N_{0}>$ $10 d B$ at a high normalized user-load of $\gamma=K / S_{f}=12 / 4$, when supporting $K=12$ users. This user-load is three times higher than the number of chips per FD spreading sequence. Furthermore, the Bayesian MUD is also capable of supporting $K=12$ users, but imposes a higher complexity than that of the proposed hybrid MUD. A comparison between the complexity and BER performance of the different MUDs supporting $K=8$ users at $E_{b} / N_{0}=6 d B$ as well as supporting $K=12$ users at $E_{b} / N_{0}=10 d B$ is listed in Table II. ${ }^{1}$ The complexity was derived by taking into account the complexity of the specific MUDs employed and their associated number of iterations, while the complexity imposed by the channel decoder may be deemed insignificant compared to that imposed by the MUD. It is clearly seen that the proposed hybrid MUD outperforms both the MF-SoIC MUD and the Bayesian MUD in terms of the complexity imposed, while maintaining a simillar BER performance to the latter.

\section{CONCLUSION}

In this paper we proposed a novel hybrid turbo MUD scheme, which combines the benefits of different MUDs, namely that of low complexity and high performance. We analyzed and compared the convergence behaviours of different MUDs With the aid of EXIT charts, and then designed a novel hybrid MUD, switching between different MUDs by monitoring the mutual information gain achieved

\footnotetext{
${ }^{1}$ It is worth noting nonetheless that according to Fig.4 at $E_{b} / N_{0}=6 d B$ the MF-SoIC and Bayesian MUDs required $I_{\text {SoIC }}=9$ amd $I_{\text {Bayesian }}=5$ iterations, respectively.
}

\begin{tabular}{|l|c|r|}
\hline \multicolumn{3}{|c|}{$K=8, E_{b} / N_{0}=6 d B$} \\
\hline Type of MUD & Complexity (Operation) & BER \\
\hline SoIC & $9 * 4 *(8-3)=180$ & $2.75 \mathrm{e}-05$ \\
\hline Bayesian & $5 * 2^{8} *(4 * 8+6)=48,640$ & $2.33 \mathrm{e}-05$ \\
\hline Hybrid & $9 * 4 *(8-3)=180$ \\
\hline \multicolumn{3}{|c|}{$K=12, E_{b} / N_{0}=10 d B$} \\
\hline Type of MUD & Complexity (Operation) & BER \\
\hline SoIC & $3 * 4 *(12-3)=108$ & 0.341 \\
\hline Bayesian & $7 * 2^{12} *(4 * 12+6)=1,548,288$ & $4.051 \mathrm{e}-06$ \\
\hline Hybrid & $5 * 4 *(12-3)+$ & $4.074 \mathrm{e}-06$ \\
\hline
\end{tabular}

TABLE II

COMPARISONS BETWEEN THE COMPLEXITY AND THE BER PERFORMANCE OF DIFFERENT MUDS.

by each iteration. Since it is not feasible to measure the mutual information directly, we proposed a realistic estimation of the mutual information gain. Fortunately, the mutual information was found to be a near-linear function of the extrinsic LLR values, when assuming Gaussian distributed LLRs at the output of the MUD. The MFSoIC MUD and the Bayesian MUD were combined in order to create an advanced hybrid MUD. The simulation results guantified the performance advantage of employing the hybrid MUD, which was capable of supporting a high normalized user-load of $\gamma=K / S_{f}=$ 12/4. At this load the MF-SoIC MUD failed to converge, whereas the proposed hybrid MUD achieved an infinitesimally low BER, which was similar to that of the Bayesian MUD, while imposing only about half the complexity.

\section{REFERENCES}

[1] P. Li, L. Liu, K. Wu, and W. K. Leung, "Interleave-division multipleaccess," IEEE Transactions on Wireless Communications, vol. 5, pp. 938947, Apr. 2006

[2] S. ten Brink, "Convergence behavior of iteratively decoded parallel concatenated codes," IEEE Transactions on Communications, vol. 49, pp. 1727-1737, Oct. 2001.

[3] L. Hanzo, M. Münster, B. Choi, and T. Keller, OFDM and MC-CDMA for Broadcasting Multi-User Communications, WLANs and Broadcasting. Wiley-IEEE Press, 2003.

[4] S. Chen, B. Mulgrew, and S. McLaughlin, "Adaptive Bayesian equaliser with decision feedback," IEEE Transactions on Signal Processing, vol. 41, pp. 2918-2927, 1993.

[5] L. Xu, S. Chen, and L. Hanzo, "Exit chart analysis of low-complexity Bayesian turbo multiuser detection for rank-deficient multiple antenna aided ofdm," in Proc. VTC 2007, (Baltimore, USA), pp. G27-31, Sept.30 - Oct 3, 2007. 